\title{
Quantification of Amine- and Alcohol-Containing Metabolites in Saline Samples Using Pre-extraction Benzoyl Chloride Derivatization and Ultrahigh Performance Liquid Chromatography Tandem Mass Spectrometry (UHPLC MS/MS)
}

Brittany Widner,* Melissa C. Kido Soule, Frank Xavier Ferrer-González, Mary Ann Moran, and Elizabeth B. Kujawinski

Cite This: Anal. Chem. 2021, 93, 4809-4817

Read Online

ACCESS | Lill Metrics \& More | 期 Article Recommendations

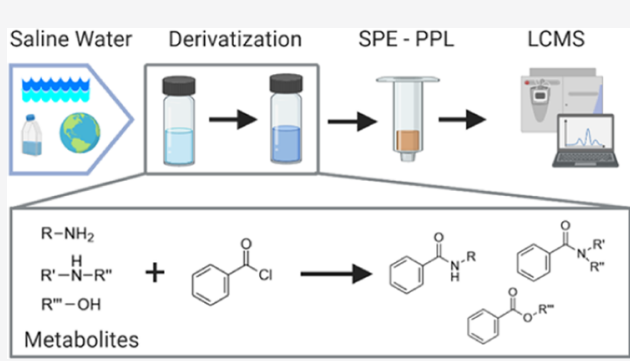

ABSTRACT: Dissolved metabolites serve as nutrition, energy, and chemical signals for microbial systems. However, the full scope and magnitude of these processes in marine systems are unknown, largely due to insufficient methods, including poor extraction of small, polar compounds using common solid-phase extraction resins. Here, we utilized pre-extraction derivatization and ultrahigh performance liquid chromatography electrospray ionization tandem mass spectrometry (UHPLC-ESI-MS/MS) to detect and quantify targeted dissolved metabolites in seawater and saline culture media. Metabolites were derivatized with benzoyl chloride by their primary and secondary amine and alcohol functionalities and quantified using stable isotope-labeled internal standards (SIL-

ISs) produced from ${ }^{13} \mathrm{C}_{6}$-labeled benzoyl chloride. We optimized derivatization, extraction, and sample preparation for field and culture samples and evaluated matrix-derived biases. We have optimized this quantitative method for 73 common metabolites, of which 50 cannot be quantified without derivatization due to low extraction efficiencies. Of the 73 metabolites, 66 were identified in either culture media or seawater and 45 of those were quantified. This derivatization method is sensitive (detection limits $=\mathrm{pM}$ to $\mathrm{nM}$ ), rapid ( $\sim 5$ min per sample), and high throughput.

\section{INTRODUCTION}

Metabolomics is the study of all molecules produced as a result of cellular metabolism in a biological or environmental system. In aquatic environments, dissolved ("extracellular") metabolites are the chemical currencies that link organisms across trophic levels and spatial and temporal scales. ${ }^{1}$ They satisfy nutritional and energetic demands of cells ${ }^{1-5}$ and promote interactions within and between taxa. ${ }^{4,6,7}$ All marine organisms, from prokaryotes ${ }^{8}$ to animals, ${ }^{9}$ release metabolites into seawater, and differential metabolite release can reflect the nutritional status as well as cell death via viral lysis, grazing, predation, and apoptosis. ${ }^{10}$ Qualitative and quantitative assessments of these processes therefore require the identification and quantification of dissolved metabolites in the seawater matrix.

Dissolved marine metabolites are challenging to quantify due to their high variability in structure and polarity. Metabolites can be bases (e.g., polyamines) or acids (e.g., carboxylic acids), exhibit negative (e.g., phosphates), positive (e.g., amines), or neutral (e.g., carbohydrates) charges, range in size from 50 to $>1000 \mathrm{amu}$, and vary in aromaticity and heteroatom composition. Metabolite analysis in seawater is particularly challenging because of high levels of inorganic salts $(\mathrm{mM})$ relative to analytes $(\mu \mathrm{M}$ to $\mathrm{fM})$. As a result, historical methods targeted groups of related molecules, including amino acids, ${ }^{11}$ the $\mathrm{B}$ vitamins, ${ }^{12}$ and sugars. ${ }^{13}$ While effective for the targeted molecules, these piecemeal approaches are time consuming, fall short for molecules that resist strict classification, and do not facilitate the identification of novel compounds.

Current methods in marine dissolved metabolomics seek to identify and quantify tens to hundreds of compounds with varying physicochemical properties in diverse sample types often using mass spectrometry (MS). ${ }^{14-16}$ Prior to MS analysis, dissolved metabolites must be extracted from seawater to remove salts. Solid-phase extraction (SPE) using the aromatic PPL resin is a common approach for isolating dissolved organic matter (DOM) from seawater ${ }^{17}$ that has been applied to dissolved metabolites. ${ }^{14,16} \mathrm{PPL}$ cartridges are commercially available and high throughput, but PPL extraction suffers from a significant bias that varies by matrix

Received: September 4, 2020

Accepted: February 26, 2021

Published: March 10, 2021 
and compound type. ${ }^{18,19}$ Compound-specific PPL extraction efficiencies in seawater and culture media range from 0 to $100 \%$, with efficiencies $<1 \%$ for most metabolites tested, particularly small polar biomolecules. ${ }^{16}$ Metabolite extraction with PPL also requires relatively large sample volumes $(>100$ $\mathrm{mL}$ for culture media and up to $10 \mathrm{~L}$ of seawater from the deep ocean, per replicate). High volume requirements and poor PPL extraction efficiencies have limited studies of dissolved metabolites in marine systems and cultures.

Liquid chromatography (LC) coupled to electrospray ionization (ESI) is commonly used to introduce metabolites into mass spectrometers, where signal intensity depends on analyte concentration and ionization efficiency. However, matrix components can inhibit (or enhance) analyte ionization efficiency, resulting in quantification errors. ${ }^{20}$ For example, many small, polar metabolites are poorly retained on widely used reversed-phase (RP) columns leading to coelution between analytes and with salts. Internal standards (ISs) can correct for matrix-related effects. The ideal IS is a stable isotopically labeled (SIL-IS) analyte analogue that experiences the same ionization environment. ${ }^{21}$ When isotopologues are not available, matrix effects can be somewhat ameliorated using matrix-matched standard curves, the standard addition method (SAM), or by normalization of samples and standards to similar, but not analyte-paired, SIL-IS. ${ }^{22}$ These approaches, however, have limitations. Matrices with sufficiently low background analyte concentrations are challenging to procure, particularly for picomolar metabolites in offshore marine systems. The SAM requires many replicates and produces less precise but sometimes more accurate results than an external or matrix-matched calibration curve. ${ }^{23}$ Finally, the use of alternate SIL-ISs may introduce biases if SIL-ISs are not carefully selected. $^{24}$

Functional group derivatization can improve analysis of elusive chemical species. Sogin et al. ${ }^{15}$ used derivatization to measure metabolites by gas chromatography (GC)-MS in a culture medium and sediment porewaters. This method uses convenient sample volumes $(1 \mathrm{~mL})$, but the detection limits (DLs) (mid to high $\mathrm{nM}$ ) are likely not sensitive enough for open waters, where metabolites may range from $\mathrm{fM}$ to low $\mathrm{nM}^{2,11}$ Other studies proposed that $\sim 95 \%$ of the human metabolome can be measured by derivatizing four "submetabolomes" of amine/phenolic alcohol, carboxylic acids, carbonyl, and alcohol functionalities with dansyl reagents. ${ }^{25}$ Derivatization can increase sensitivity by enhancing ionization efficiency and improving chromatographic retention and separation. ${ }^{26,27}$ Derivatization can improve absolute and relative quantification by creating analyte-paired SIL-IS. ${ }^{28}$ Aqueous derivatization protocols exist for amine groups, ${ }^{27}$ but comparable protocols for alcohols are rare, as many alcohol derivatization reagents react explosively with water. ${ }^{29}$ Benzoyl chloride (BC) can derivatize (benzoylate) amines (primary and secondary) and alcohol groups into amides and esters, respectively, via a base-catalyzed Schotten-Baumann reaction (Figure S1). BC-based derivatization has been applied widely in biochemical and environmental settings. ${ }^{30-42} \mathrm{BC}$ reacts quickly in aqueous and organic solution at room temperature and is available in isotopically labeled forms, enabling the use of paired SIL-IS. Benzoyl derivatives are stable in cold storage and in the light $^{40}$ and can be detected with ultrahigh performance liquid chromatography (UV-HPLC), LCMS, and GC-MS. For molecules with multiple derivatizable functional groups, BC methods can produce single- or multilabeled derivatives by modification of derivatization ${ }^{37}$ or extraction ${ }^{34}$ procedures.

Here, we present a method that pairs BC derivatization with SPE-PPL and UHPLC-ESI-MSMS to quantify small polar metabolites in seawater and saline culture media containing primary and secondary amine and alcohol functionalities (Figure 1). We optimized $\mathrm{pH}$, reagent composition and

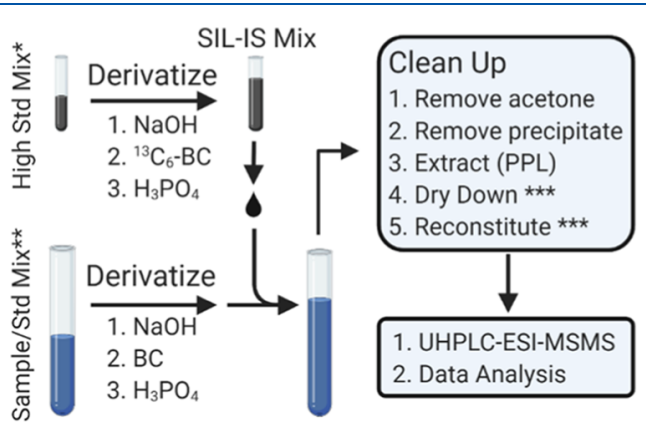

Figure 1. Method overview. *The standard mix for SIL-ISs is prepared in the same matrix as the standard curve. **Standard (Std) mixes for the calibration curve are prepared immediately prior to derivatization. Standards (for SIL-IS and calibration curve) and samples are derivatized simultaneously, and identical aliquots of the SIL-IS mix are added to samples and calibration standards following $\mathrm{H}_{3} \mathrm{PO}_{4}$ addition. ***Dry down and reconstitution include "washing" to remove precipitate in sample volumes $\geq 10 \mathrm{~mL}$.

concentration, buffer type, and extraction for saline samples, with analyte-paired SIL-IS produced from ${ }^{13} \mathrm{C}_{6}$-labeled BC. We quantified matrix-derived biases (process effects) for 73 metabolites and established their detection limits, from $\mathrm{pM}$ to $\mathrm{nM}$. This method is high throughput because the initial sample processing is fast $(5 \mathrm{~min}$ per sample when running batches) and requires low sample volumes (1-25 mL, depending on the sample type). BC derivatization improves chromatographic performance, relative to methods without derivatization. Together, these improvements expand the suite of marine dissolved metabolites accessible by LCMS.

\section{EXPERIMENTAL SECTION}

Chemicals and Standards. Hydrochloric acid $(\mathrm{HCl})$, acetone, methanol, acetonitrile (ACN), and formic acid were purchased from Fisher Scientific (Optima), as were BC (99\%, ACROS Organics) and sodium hydroxide $(\mathrm{NaOH})$. Phosphoric acid (85\%, ACS reagent grade) was purchased from Sigma-Aldrich. SIL-IS derivatives were prepared with either $\mathrm{D}_{5}$-BC $\left(99 \%\right.$ atom D) or ${ }^{13} \mathrm{C}_{6}$-ring-BC $\left(99 \%\right.$ atom $\left.{ }^{13} \mathrm{C}\right)$ purchased from Cambridge Isotopes and Sigma-Aldrich, respectively. Standards were purchased from Sigma-Aldrich except for 2,3-dihydroxypropane-1-sulfonate (DHPS) obtained by custom synthesis. Deionized (DI) water was obtained from a Milli-Q system (Millipore; resistivity $18.2 \mathrm{M} \Omega$ at $24{ }^{\circ} \mathrm{C}$, TOC $<1 \mu \mathrm{M})$. Samples and reagents were stored in acidwashed, combusted (at least $4 \mathrm{~h}$ at $450{ }^{\circ} \mathrm{C}$ ), or solvent-rinsed ( $3 \times$ each methanol, acetone, dichloromethane) glassware. BC and all solvents were transferred using solvent-rinsed glass syringes with metal needles (Hamilton). Primary stocks and mixes were stored at $-20{ }^{\circ} \mathrm{C}$. The working reagent $(5 \% \mathrm{BC}$ in acetone) was prepared fresh daily.

Sample Collection. Filtered $(0.2 \mu \mathrm{m}$, poly(tetrafluoroethylene) (PTFE)) seawater collected from two locations (Vineyard Sound, MA, and the Bermuda Atlantic 
Table 1. Method Parameters and Detection Limits for Benzoylated Metabolites

\begin{tabular}{|c|c|c|c|c|c|c|c|c|}
\hline \multicolumn{6}{|c|}{ LCMS Parameters } & \multicolumn{3}{|c|}{ Detection Limit (nM) } \\
\hline Compound & Polarity & $\mathrm{RT}(\min )$ & $\begin{array}{l}\text { Precursor } \\
(\mathrm{m} / z)\end{array}$ & Product $1(m / z)$ & Product $2(m / z)$ & BATS & $\begin{array}{c}T . \\
\text { pseudonana }\end{array}$ & R. pomeroyi \\
\hline (6R)-5,6,7,8-tetrahydrobiopterin ${ }^{1}$ & + & $2.7 *$ & 346.1510 & 105.0330 & 270.0964 & $\mathrm{NaN}$ & $3.6^{\mathrm{b}}$ & $22^{\mathrm{a}}$ \\
\hline $2^{\prime}$ deoxycitidine $^{1}$ & - & $7.7 *$ & 330.1095 & 197.0703 & 287.1306 & $0.14^{\mathrm{a}}$ & $19^{\mathrm{a}}$ & $\mathrm{NaN}$ \\
\hline $2^{\prime}$ deoxyguanosine $e^{1}$ & - & $8.5^{*}$ & 370.1157 & 121.0281 & 150.0406 & $0.052^{\mathrm{a}}$ & $0.85^{\mathrm{a}}$ & $0.68^{\mathrm{b}}$ \\
\hline $2^{\prime}$ deoxyuridine ${ }^{1}$ & - & $8.5^{*}$ & 331.0935 & 136.8930 & 121.0290 & $\mathrm{NaN}$ & $3.9^{\mathrm{b}}$ & $21^{\mathrm{a}}$ \\
\hline $3^{\prime} \mathrm{AMP}^{1, *}$ & + & 5 & 452.0966 & 105.0330 & 136.0618 & $0.0091^{\mathrm{b}}$ & $0.088^{\mathrm{a}}$ & $0.45^{\mathrm{a}}$ \\
\hline 4-aminobenzoic acid ${ }^{1}$ & + & 7.8 & 242.0812 & 105.0328 & $\mathrm{NaN}$ & $0.14^{\mathrm{a}}$ & $22^{\mathrm{a}}$ & $0.91^{\mathrm{a}}$ \\
\hline $5^{\prime} \mathrm{UMP}^{1, *}$ & - & 6.5 & 427.0548 & 78.9586 & $\mathrm{NaN}$ & $0.018^{\mathrm{a}}$ & $18^{\alpha, \mathrm{a}}$ & $3.1^{\alpha, \mathrm{b}}$ \\
\hline $5^{\prime} \mathrm{AMP}^{1, *}$ & - & 6.7 & 450.0820 & 134.0456 & 78.9579 & $0.034^{\mathrm{a}}$ & $26^{\alpha, \mathrm{a}}$ & $18^{\alpha, \mathrm{a}}$ \\
\hline $5^{\prime}$ deoxyadenosine ${ }^{1, *}$ & - & 6.1 & 354.1208 & 238.0727 & $\mathrm{NaN}$ & $\mathrm{NaN}$ & $12^{\mathrm{a}}$ & $\mathrm{NaN}$ \\
\hline adenine $^{1, *}$ & + & 7.4 & 240.0880 & 105.0328 & 137.0449 & $0.17^{\alpha, \mathrm{a}}$ & $18^{\mathrm{a}}$ & $13^{\mathrm{b}}$ \\
\hline adenosine $^{0}$ & + & 1.7 & 268.1042 & 136.0615 & $\mathrm{NaN}$ & $0.16^{\mathrm{a}}$ & $28^{\mathrm{a}}$ & $26^{\mathrm{a}}$ \\
\hline alanine $e^{1, *}$ & - & 6.6 & 192.0666 & 148.0762 & 120.0443 & $0.069^{b}$ & $22^{\alpha, \mathrm{b}}$ & $16^{\mathrm{a}}$ \\
\hline $\operatorname{amMP}^{1, *}$ & + & 3.7 & 243.1240 & 122.0714 & 81.0445 & $0.030^{\mathrm{a}}$ & $11^{\mathrm{b}}$ & $9.3^{\mathrm{b}}$ \\
\hline $\operatorname{arginin} e^{1, *}$ & - & 3.3 & 277.1306 & 191.1167 & 120.0443 & $0.065^{\mathrm{a}}$ & $8.4^{\mathrm{a}}$ & $0.95^{\mathrm{b}}$ \\
\hline asparagine $^{1, *}$ & - & 3.4 & 235.0724 & 114.0182 & 96.0086 & $0.041^{\mathrm{a}}$ & $2.1^{\mathrm{b}}$ & $0.60^{\mathrm{b}}$ \\
\hline aspartate ${ }^{1 *}$ & + & 4.5 & 238.0710 & 105.0328 & 95.0484 & $0.24^{\mathrm{a}}$ & $1.3^{\mathrm{b}}$ & $1.2^{\mathrm{b}}$ \\
\hline chitobiose $^{1, *}$ & + & $7.0 *$ & 529.2028 & 126.0543 & 204.0860 & $\mathrm{NaN}$ & $14^{\mathrm{a}}$ & $35^{\mathrm{a}}$ \\
\hline chitotriose $\mathrm{e}^{1}$ & + & $7.0 *$ & 732.2822 & 126.0543 & 138.0550 & $0.016^{\mathrm{a}}$ & $22^{\mathrm{a}}$ & $0.44^{\alpha, \mathrm{a}}$ \\
\hline ciliatine $^{1, *}$ & + & 3 & 230.0577 & 105.0328 & 95.0484 & $0.016^{\mathrm{b}}$ & $0.035^{b}$ & $0.15^{\mathrm{b}}$ \\
\hline citrulline $^{1, *}$ & + & 4.6 & 280.1292 & 105.0328 & 113.0702 & $0.079^{\mathrm{a}}$ & $1.3^{\mathrm{b}}$ & $1.2^{\mathrm{a}}$ \\
\hline cysteate $^{1, *}$ & - & 2.8 & 272.0234 & 149.9958 & 80.9645 & $0.019^{\mathrm{a}}$ & $0.42^{\mathrm{a}}$ & $0.63^{\mathrm{b}}$ \\
\hline cysteine $^{1, \gamma}$ & + & 11.2 & 449.0846 & 105.0330 & 224.0368 & $0.018^{\mathrm{b}}$ & $0.38^{\mathrm{b}}$ & $1.1^{\mathrm{a}}$ \\
\hline cytidine $^{1}$ & + & $7.1^{*}$ & 348.1190 & 105.0330 & 216.0765 & $0.045^{\mathrm{a}}$ & $26^{\mathrm{a}}$ & $\mathrm{NaN}$ \\
\hline cytosine $\mathrm{e}^{1, *}$ & + & 7.4 & 216.0767 & 105.0328 & 95.0484 & $\mathrm{NaN}$ & $67^{\mathrm{a}}$ & $20^{\mathrm{b}}$ \\
\hline desthiobiotin $^{0}$ & + & 7.3 & 215.1390 & 179.1161 & 197.1281 & $\mathrm{NaN}$ & $0.67^{\alpha, \mathrm{b}}$ & $0.89^{\alpha, \mathrm{a}}$ \\
\hline DHPS $^{1, *}$ & - & 5.4 & 259.0282 & 121.0285 & $\mathrm{NaN}$ & $0.0056^{\mathrm{b}}$ & $0.49^{\mathrm{a}}$ & $0.12^{\mathrm{b}}$ \\
\hline ectoine $\mathrm{e}^{1, *, \gamma}$ & + & 5.4 & 265.1187 & 105.0330 & 98.0596 & $0.077^{\mathrm{a}}$ & $8.4^{\mathrm{a}}$ & $40^{\mathrm{a}}$ \\
\hline Folate $^{1}$ & - & 7.5 & 544.1586 & 325.1192 & 240.0646 & $\mathrm{NaN}$ & $0.13^{\alpha, \mathrm{b}}$ & $4.1^{\alpha, \mathrm{b}}$ \\
\hline $\mathrm{GABA}^{1, *}$ & + & 6.8 & 208.0968 & 105.0328 & 95.0484 & $0.041^{\mathrm{a}}$ & $0.22^{\mathrm{b}}$ & $0.15^{\mathrm{b}}$ \\
\hline glucosamine-6-phosphate ${ }^{1, *}$ & - & 2.4 & 362.0646 & 78.9579 & 96.9681 & $\mathrm{NaN}$ & $1.4^{\alpha, \mathrm{a}}$ & $5.0^{\mathrm{b}}$ \\
\hline glutamic acid ${ }^{1, *}$ & + & 5.5 & 252.0866 & 105.0328 & 84.0440 & $0.095^{\mathrm{a}}$ & $2.2^{\mathrm{b}}$ & $4.2^{\mathrm{b}}$ \\
\hline glutamine $e^{1, *}$ & - & 3.8 & 249.0881 & 120.0443 & 187.0880 & $0.067^{\mathrm{a}}$ & $1.3^{\mathrm{b}}$ & $0.48^{\mathrm{a}}$ \\
\hline glycine $^{1, *}$ & - & 4.3 & 178.0510 & 132.0438 & 134.0607 & $0.051^{\mathrm{a}}$ & $87^{\mathrm{a}}$ & $0.16^{\mathrm{b}}$ \\
\hline glyphosate $^{1}$ & - & 2.1 & 272.0329 & 149.9945 & 62.9635 & $\mathrm{NaN}$ & $5.3^{\mathrm{b}}$ & $1.6^{\mathrm{b}}$ \\
\hline guanine $^{1, *}$ & + & 7.5 & 256.0829 & 105.0328 & 238.0705 & $\mathrm{NaN}$ & $62^{\mathrm{a}}$ & $73^{\alpha, \mathrm{a}}$ \\
\hline guanosine $e^{1}$ & + & 8.2 & 388.1251 & 105.0328 & 152.0556 & $0.019^{\mathrm{b}}$ & $0.56^{\mathrm{b}}$ & $14^{\mathrm{a}}$ \\
\hline $\mathrm{HET}^{1}$ & + & 12.3 & 248.0740 & 188.0352 & 105.0328 & $0.30^{\mathrm{a}}$ & $52^{\mathrm{a}}$ & $51^{a}$ \\
\hline histidine $^{1}$ & - & 2.7 & 258.0884 & 120.0443 & 81.0452 & $0.14^{\mathrm{a}}$ & $12^{\mathrm{a}}$ & $0.94^{\mathrm{a}}$ \\
\hline $\mathrm{HMP}^{1, *}$ & + & 6.3 & 244.1080 & 105.0330 & 123.0456 & $0.016^{\mathrm{b}}$ & $0.33^{\mathrm{a}}$ & $\mathrm{NaN}$ \\
\hline homoserine $e^{1, *}$ & - & 4.2 & 222.0772 & 120.0449 & 178.0872 & $0.051^{\mathrm{b}}$ & $0.12^{\mathrm{b}}$ & $1.2^{\mathrm{a}}$ \\
\hline homoserine betaine $e^{1, *}$ & + & 5 & 266.1387 & 105.0330 & 207.0643 & $\mathrm{NaN}$ & $0.92^{\mathrm{b}}$ & $1.9^{\mathrm{b}}$ \\
\hline inosine $^{1}$ & - & 7.6 & 371.0997 & 121.0289 & 177.0408 & $0.043^{\mathrm{a}}$ & $0.42^{\mathrm{a}}$ & $19^{\mathrm{a}}$ \\
\hline isethionate $^{1, *}$ & - & 6.4 & 229.0176 & 121.0289 & $\mathrm{NaN}$ & $0.040^{\mathrm{a}}$ & $0.26^{\mathrm{a}}$ & $0.076^{\mathrm{b}}$ \\
\hline isoleucine $^{1}$ & - & 11.4 & 234.1136 & 190.1247 & 82.0660 & $0.050^{\mathrm{a}}$ & $7.8^{\mathrm{a}}$ & $0.61^{\mathrm{a}}$ \\
\hline kynurenine $^{1}$ & - & 11.2 & 311.1037 & 128.0492 & 144.0453 & $0.015^{\mathrm{a}}$ & $1.5^{\mathrm{b}}$ & $5.2^{\mathrm{b}}$ \\
\hline leucine $e^{1}$ & - & 11.2 & 234.1136 & 190.1219 & 82.0652 & $0.042^{\mathrm{a}}$ & $1.9^{\mathrm{a}}$ & $0.28^{\mathrm{b}}$ \\
\hline lysine $\mathrm{e}^{2, *}$ & - & 11 & 353.1507 & 120.0443 & 188.1060 & $0.038^{\mathrm{a}}$ & $3.4^{\mathrm{b}}$ & $5.9^{\mathrm{a}}$ \\
\hline malic acid $^{1, *}$ & - & 7.8 & 237.0404 & 121.0290 & 70.0130 & $0.044^{\mathrm{b}}$ & $27^{\alpha, \mathrm{a}}$ & $1.7^{\alpha, \mathrm{a}}$ \\
\hline methionine $e^{1, *}$ & + & 9.4 & 254.0845 & 160.0762 & 105.0329 & $\mathrm{NaN}$ & $\mathrm{NaN}$ & $25^{\mathrm{a}}$ \\
\hline $\mathrm{MTA}^{0, \gamma}$ & + & $1.6^{*}$ & 314.0918 & 136.0616 & 97.0282 & $\mathrm{NaN}$ & $1.2^{\alpha, \mathrm{a}}$ & $\mathrm{NaN}$ \\
\hline muramic acid ${ }^{1, *, \gamma}$ & + & $6.4^{*}$ & 338.1239 & 105.0330 & 200.0704 & $0.051^{\mathrm{b}}$ & $0.81^{\mathrm{a}}$ & $0.15^{\mathrm{a}}$ \\
\hline$N$-acetyl-D-glucosamine $e^{1, *}$ & + & $7.1 *$ & 326.1234 & 126.0539 & 105.0330 & $\mathrm{NaN}$ & $0.43^{\alpha, \mathrm{a}}$ & $9.3^{\alpha, \mathrm{a}}$ \\
\hline$N$-acetyl-muramic acid ${ }^{1, \gamma}$ & + & $8.1^{*}$ & 380.1338 & 105.0330 & 126.0543 & $0.047^{\mathrm{a}}$ & $0.23^{\mathrm{a}}$ & $8.0^{\mathrm{a}}$ \\
\hline ornithine $e^{2, *}$ & + & 10.4 & 341.1496 & 105.0330 & 174.0911 & $0.081^{\mathrm{a}}$ & $1.5^{\mathrm{b}}$ & $0.79^{\mathrm{a}}$ \\
\hline pantothenic acid ${ }^{1}$ & - & 10.8 & 322.1296 & 121.0289 & 128.0711 & $0.0015^{\mathrm{b}}$ & $0.086^{\mathrm{a}}$ & $0.014^{\mathrm{b}}$ \\
\hline phenylalanine $^{1}$ & + & 11.7 & 270.1125 & 105.0328 & 120.0800 & $0.069^{\mathrm{a}}$ & $0.68^{\mathrm{b}}$ & $0.15^{\mathrm{b}}$ \\
\hline proline $^{1, *}$ & - & 7.8 & 218.0823 & 146.0608 & 172.0766 & $0.085^{\mathrm{a}}$ & $4.2^{\mathrm{b}}$ & $1.8^{\mathrm{a}}$ \\
\hline putrescine $\mathrm{e}^{2, *}$ & - & 11.2 & 295.1452 & 120.0442 & 174.0911 & $0.0078^{b}$ & $0.38^{\mathrm{a}}$ & $0.096^{\mathrm{a}}$ \\
\hline pyridoxine $e^{1}$ & + & 7.3 & 274.1074 & 105.0330 & 256.0957 & $\mathrm{NaN}$ & $0.33^{\mathrm{a}}$ & $22^{\mathrm{a}}$ \\
\hline
\end{tabular}


Table 1. continued

\begin{tabular}{|c|c|c|c|c|c|c|c|c|}
\hline \multicolumn{6}{|c|}{ LCMS Parameters } & \multicolumn{3}{|c|}{ Detection Limit (nM) } \\
\hline Compound & Polarity & $\mathrm{RT}(\min )$ & $\begin{array}{l}\text { Precursor } \\
(m / z)\end{array}$ & Product $1(m / z)$ & Product $2(m / z)$ & BATS & $\begin{array}{c}T . \\
\text { pseudonana }\end{array}$ & R. pomeroyi \\
\hline$S$-(1,2-dicarboxyethyl) glutathione $e^{1, *}$ & + & 6.5 & 528.1282 & 192.0318 & 105.0330 & $0.00095^{\alpha, \mathrm{b}}$ & $\mathrm{NaN}$ & $8.4^{\alpha, \mathrm{a}}$ \\
\hline$S$-(5'adenosyl)-L-homocysteine ${ }^{0, \gamma}$ & + & 3.4 & 505.1511 & 136.0611 & 97.0279 & $\mathrm{NaN}$ & $\mathrm{NaN}$ & $18^{\alpha, \mathrm{a}}$ \\
\hline $\operatorname{sarcosine} e^{1, *}$ & - & 6.2 & 192.0666 & 131.0372 & 146.0608 & $0.081^{\mathrm{a}}$ & $0.43^{\mathrm{a}}$ & $0.38^{\mathrm{a}}$ \\
\hline serine $e^{1, *}$ & + & 3.7 & 210.0761 & 105.0328 & 95.0485 & $\mathrm{NaN}$ & $12^{\mathrm{b}}$ & $27^{\mathrm{a}}$ \\
\hline spermidine $^{3, *}$ & + & 12.7 & 458.2438 & 162.0911 & 336.2064 & $0.014^{\mathrm{b}}$ & $0.10^{\mathrm{b}}$ & $0.14^{\mathrm{a}}$ \\
\hline taurine $e^{1, *}$ & - & 3.3 & 228.0336 & 106.9800 & 79.9564 & $0.042^{\mathrm{a}}$ & $0.58^{\mathrm{a}}$ & $0.38^{\mathrm{a}}$ \\
\hline taurocholic acid ${ }^{0}$ & - & 12 & 514.2844 & 514.2844 & $\mathrm{NaN}$ & $\mathrm{NaN}$ & $0.41^{\alpha, \mathrm{b}}$ & $0.049^{\alpha, \mathrm{b}}$ \\
\hline threonine $\mathrm{e}^{1, *}$ & + & 5.5 & 224.0917 & 105.0329 & 56.0491 & $0.15^{\mathrm{a}}$ & $0.77^{\mathrm{b}}$ & $0.99^{\mathrm{b}}$ \\
\hline tryptamine $e^{1}$ & + & 12.7 & 265.1335 & 105.0330 & 144.0798 & $0.00044^{\mathrm{b}}$ & $71^{\alpha, \mathrm{a}}$ & $0.38^{\mathrm{a}}$ \\
\hline tryptophan ${ }^{1}$ & - & 11.4 & 307.1088 & 120.0442 & 134.0606 & $0.040^{\mathrm{a}}$ & $12^{\mathrm{a}}$ & $0.42^{\mathrm{a}}$ \\
\hline tyrosine $^{1, *}$ & - & 8.4 & 284.0928 & 119.0492 & 120.0442 & $0.17^{\mathrm{a}}$ & $15^{\alpha, \mathrm{a}}$ & $6.0^{\mathrm{b}}$ \\
\hline uridine $e^{1, *}$ & - & 8.1 & 347.0885 & 110.0243 & 121.0289 & $0.014^{\mathrm{a}}$ & $0.77^{\mathrm{b}}$ & $0.25^{\mathrm{b}}$ \\
\hline valine $^{1, *}$ & - & 9.4 & 220.0979 & 176.1062 & 68.0495 & $0.10^{\mathrm{a}}$ & $6.4^{\mathrm{b}}$ & $0.71^{\mathrm{a}}$ \\
\hline xanthosine $\mathrm{e}^{1}$ & - & 8.5 & 387.0946 & 151.0247 & 193.0350 & $0.049^{\mathrm{a}}$ & $9.0^{\mathrm{a}}$ & $31^{\alpha, \mathrm{a}}$ \\
\hline
\end{tabular}

${ }^{a} \gamma$ Compounds that were not detected as $[\mathrm{M}+\mathrm{H}]^{+}$or $[\mathrm{M}-\mathrm{H}]^{-}: \mathrm{S}-\left(5^{\prime}\right.$ adenosyl $)$-L-homocysteine, $+\mathrm{OH}$; cysteine, dimer $(+\mathrm{H})$; ectoine gain $\mathrm{H}_{2} \mathrm{O}$ $( \pm \mathrm{H})$; muramic acid lose $\mathrm{H}_{2} \mathrm{O}(+\mathrm{H}) ; 5^{\prime}$-deoxy-5'-methylthioadenosine oxidized $(+\mathrm{H})$; $n$-acetyl-muramic acid lose $\mathrm{H}_{2} \mathrm{O}(+\mathrm{H})$. $*$ Compounds that cannot be quantified following PPL extraction without derivatization. ${ }^{16} 0,1,2,3$ The number of benzoyl labels on the compound $*$ on retention time (RT) indicates compounds with multiple distinct peaks. In that case, RT is the maximum of the dominant peak. ${ }^{\mathrm{a}}$ and ${ }^{\mathrm{b}}$ indicate DLs calculated for the homoscedastic ${ }^{50}$ and heteroscedastic ${ }^{48}$ cases, respectively. NaN indicates that the DL could not be calculated due to an inadequate standard curve $\left(R^{2}<0.9\right)^{\alpha}$ indicates DL calculated from the raw peak area. All other DLs were calculated from the SIL-IS normalized peak area. Parameters in this table produced the most sensitive detection for each compound. Parameters for the suboptimal ionization mode and number of benzoyl groups are in Table S5.

Time-series Study site (BATS)) and spent media from Thalassiosira pseudonana and Ruegeria pomeroyi cultures was used. Details on sample collection, culturing, and experimental design are in the Supporting Text(S-Text).

Derivatization. All filtered samples were basified with a solution of $8 \mathrm{M} \mathrm{NaOH}$ and derivatized by the addition of the working $\mathrm{BC}$ reagent. Because $\mathrm{BC}$ is insoluble in water, insoluble "beads" form when added to aqueous samples, and derivatization occurs at the surface of these beads. Samples were vigorously mixed for $5 \mathrm{~min}$ (vortex for spent media and repeated inversions for field samples) or until the BC beads disappeared. The samples were then acidified with concentrated phosphoric acid and stored at $-20{ }^{\circ} \mathrm{C}$. For $1 \mathrm{~mL}$ of spent medium samples, $30 \mu \mathrm{L}$ of $\mathrm{NaOH}, 200 \mu \mathrm{L}$ of working reagent, and $15 \mu \mathrm{L}$ of phosphoric acid were added, while for 25 $\mathrm{mL}$ of seawater samples, $750 \mu \mathrm{L}$ of $\mathrm{NaOH}, 5 \mathrm{~mL}$ of working reagent, and $375 \mu \mathrm{L}$ of phosphoric acid were added.

Postderivatization Sample Prep. We removed acetone prior to extraction on the Bond Elut PPL cartridge (Agilent) to minimize its interference with resin adsorption of the derivatives (culture samples in $4 \mathrm{~mL}$ vials with vacufuge; field samples in $40 \mathrm{~mL}$ vials with $\mathrm{N}_{2}$ gas). The change in sample mass was used to confirm acetone removal, and acetone was replaced with the equivalent volume of DI water. Upon acetone removal, a large amount of white precipitate formed, which we separated from the liquid by centrifugation at low speed (1000 rcf for $15 \mathrm{~min}$ ) to avoid glass breakage. Either $1 \mathrm{~mL}$ (cultures) or $25 \mathrm{~mL}$ (field) of the resulting liquid was transferred onto a preconditioned $(6 \mathrm{~mL}$ of methanol followed by $24 \mathrm{~mL}$ of $0.01 \mathrm{M} \mathrm{HCl}) 1 \mathrm{~g} / 6 \mathrm{~mL}$ of PPL cartridge and gravity-loaded. Samples were eluted by gravity with 1 cartridge volume of methanol. The eluent was evaporated to near-dryness in a vacufuge, resulting in the formation of a white precipitate. Culture samples were immediately reconstituted in 5\% ACN/DI, but, due to the higher BC addition, the precipitate in field samples did not completely dissolve in
$5 \% \mathrm{ACN} / \mathrm{DI}$, and so, these samples received additional washing and centrifugation. Specifically, we added $500 \mu \mathrm{L}$ of $5 \% \mathrm{ACN} / \mathrm{DI}$ to each field sample, vortexed them to distribute the precipitate, and then centrifuged them (1000 rcf for 15 $\mathrm{min})$. Supernatants were transferred to $2 \mathrm{~mL}$ of glass vials for vacufuge evaporation $\left(30^{\circ} \mathrm{C}\right.$ for $\left.2 \mathrm{~h}\right)$ and then reconstituted in $5 \% \mathrm{ACN} / \mathrm{DI}$. All reconstituted samples were stored at $4{ }^{\circ} \mathrm{C}$ in $2 \mathrm{~mL}$ of LC vials with inserts, after the addition of $5 \mu \mathrm{L}$ of $\mathrm{ACN}$ to prevent precipitation during storage. Derivatization and sample preparation protocols, including pictures and video clips, are available online (dx.doi.org/10.17504/protocols.io.biukkeuw).

Standard Preparation. Metabolite concentrations were determined using matrix-matched calibration curves normalized with analyte-paired SIL-IS. A metabolite standard mix was spiked at varying levels into axenic L1 medium and $1000 \mathrm{~m}$ of BATS water for cultures and seawater samples, respectively, and then derivatized in parallel with the samples. SIL-IS mixes were prepared by adding standards to these matrices and derivatizing with ${ }^{13} \mathrm{C}_{6}$-BC. Standards and samples received the same SIL-IS addition for a given experiment immediately post derivatization. For seawater and DI water, duplicate standard curves were prepared from 5 to $1000 \mathrm{pg} / \mathrm{mL}$, each with a 200 $\mathrm{pg} / \mathrm{mL}$ SIL-IS addition. Preliminary analysis of culture samples indicated a wide range of metabolite concentrations, so we prepared two standard curves in each culture matrix, from 0 to 1000 and 1000 to $60000 \mathrm{pg} / \mathrm{mL}$, with SIL-IS additions of 100 and $10000 \mathrm{pg} / \mathrm{mL}$, respectively. Two sets of samples for each culture were prepared, receiving either the low or high SIL-IS addition. The standard addition method (SAM) was tested at BATS using samples from 1,95, 160, and $200 \mathrm{~m}$, with standard additions of $0,5,10,20,50$, and $100 \mathrm{pg} / \mathrm{mL}$, each with a 200 $\mathrm{pg} / \mathrm{mL}$ SIL-IS addition. Standard curves were prepared using the SAM in spent media from $T$. pseudonana and $R$. pomeroyi and in each axenic medium blank. See Table S1 for concentrations in $\mathrm{nM}$. 
UHPLC-ESI-MSMS and Peak ID. Samples were analyzed with a Vanquish UHPLC system (Thermo Fisher Scientific) coupled via heated electrospray ionization (H-ESI) to an ultrahigh resolution tribrid mass spectrometer, the Orbitrap Fusion Lumos (Thermo Fisher Scientific). The Lumos was operated in full MS mode with data-dependent tandem MS, guided by a list of user-defined parent ions with a retention time (RT) window (full MS/ddMS 2 with inclusion list). All data were collected in the orbitrap analyzer; MS data were used for detection and quantification, and MS/MS data were used for identification and confirmation. Detailed instrument parameters are located in the Supporting Text.

Analyte and SIL-IS peaks were integrated using Skyline, ${ }^{43,44}$ and target compounds were identified based on the correspondence of precursor $\mathrm{m} / z$, retention time, and at least one unique $\mathrm{MS}_{2}$ fragment determined from a known derivatized standard (Table 1). Metabolites were quantified from the primary adduct of the precursor, which was typically $[\mathrm{M}+\mathrm{H}]^{+}$or $[\mathrm{M}-\mathrm{H}]^{-}$for positive and negative polarities, respectively (Table 1). All subsequent calculations were performed using MATLAB (R2018b, MathWorks).

Calculations. Calibration curves were inspected manually for nonlinearity and outliers. Higher standard concentrations ( $\geq \sim 30000 \mathrm{pg} / \mathrm{mL}$ ) sometimes caused ion suppression/ enhancement of or by the SIL-IS (Figure S2) and were excluded from the curve. Only matrix-matched curves with at least five concentrations were retained. Analyte concentrations were calculated from SIL-IS normalized curves using raw peak areas normalized to analyte-matched SIL-IS peak areas. If the normalized area exhibited ion suppression/enhancement or the SIL-IS peak was too small, raw peak areas (and nonnormalized standard curves) were used (Table 1). Standard curves were required to have an $R^{2}$ greater than 0.9 for quantification and between 0.8 and 0.9 for estimation. For the SAM method, curves with at least three concentrations were retained. Analyte concentrations and standard deviations were determined by extrapolating the $x$-intercept from the linear curve fit of the standard additions and from the confidence interval of the $x$-intercept, respectively. ${ }^{23,45}$ For matrixmatched standard curves, concentrations were adjusted for nonzero analyte signals in the matrix used to prepare the standard curve.

Detection limits (DLs) were defined as the concentration at which the probability of a false positive was $5 \% .{ }^{46}$ This probability underlies the common definition of limit of detection (LOD $=3 \times$ standard deviation of a standard $\leq$ $10 \times$ of the blank), ${ }^{47}$ but that definition assumes that the standard curve residuals are not correlated with the standard concentration (homoscedastic) and requires a matrix-matched blank with negligible analyte concentrations. ${ }^{23}$ Our data rarely satisfy the latter requirement and satisfy the former only for some metabolites, thus requiring alternative DL calculations. We measured the standard deviation of normalized and raw peak areas for triplicate standards for four concentrations (Figure S3). If the standard deviation was linearly correlated with concentration (heteroscedastic, $R^{2}>0.70$ ), we calculated the DL by extrapolating the standard deviation to zero using a linear curve fit. ${ }^{48,49}$ If the standard deviation was homoscedastic $\left(R^{2}<0.7\right)$, we calculated the DL from the variability of the standard curve. ${ }^{50}$ In both cases, we assumed triplicate samples $(m=3)$.

We conducted experiments for method optimization and evaluated the effect of different matrices on quantification (process effect, PE). Experimental details are in the Supporting Text. Raw peak areas ranged by orders of magnitude, so we introduced a method performance (MP) parameter to compare treatments within an experiment. MP was calculated for each metabolite as the raw peak area $\left(\right.$ area $\left._{i}\right)$ less the mean raw peak area for all treatments $(\overline{\text { area }})$ normalized to the range of peak areas for all treatments. We multiplied this number by 2 to force the MP to range from -1 to 1 when data are symmetrical around the mean $($ mean $=0$; eq 1$)$. The median of all metabolite MPs was used to compare aggregate differences within experiments

$$
\mathrm{MP}=\frac{\text { area }_{\mathrm{i}}-\overline{\mathrm{area}}}{\max (\text { area })-\min (\text { area })} \times 2
$$

For each metabolite, PE was calculated using a modified matrix effect calculation ${ }^{51}$ (eq 2, where $X$ and "Ref" are the sample and reference matrices, respectively). PE was determined for raw peak areas as well as SIL-IS normalized peak areas, where the former reflects matrix-specific biases from the entire process (derivatization efficiency, storage, solubility, extraction efficiencies, and chromatographic and ionization effects) and the latter reflects matrix-specific biases upstream of SIL-IS addition (derivatization efficiency). A PE of zero indicates no effect, while a positive or negative $\mathrm{PE}$ indicates enhancement or reduction of the signal relative to a reference matrix. $\mathrm{PE}$ was calculated using standard curves from 0 to $100 \mathrm{pg} / \mathrm{mL}(0,5$, $10,20,50$, and $100 \mathrm{pg} / \mathrm{mL}$ ) for BATS seawater and from 3500 to $30000 \mathrm{pg} / \mathrm{mL}$ (3500, 7000, 10000 , and $30000 \mathrm{pgmL})$ for culture media. The reference matrices for seawater and cultures were seawater from $1000 \mathrm{~m}$ and L1 medium, respectively

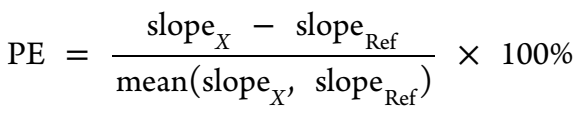

\section{RESULTS AND DISCUSSION}

We optimized derivatization, extraction, and instrument parameters to quantify a large number of compounds with high accuracy and sensitivity. Our method optimization prioritized reduced sample volumes, decreased per-sample costs, and increased sample throughput. We used differences in $\mathrm{MP}$ to determine the ideal buffer type, solvent, $\mathrm{pH}$, and $\mathrm{BC}$ concentrations. For method validation, we evaluated metabolites in both ionization modes (Figures 2 and S4-S12; Tables S2-S4).

Alcohol and amine functionalities require slightly different reaction conditions for optimal recovery of benzoyl deriva-

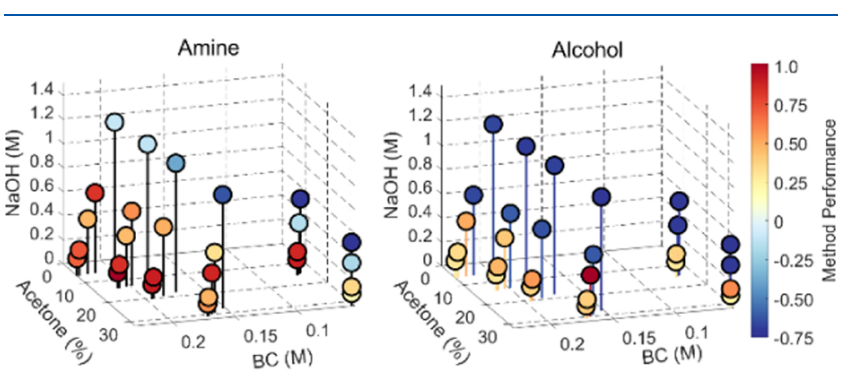

Figure 2. Effect of reagent concentrations on mean method performance (MP) in seawater for compounds with primary or secondary amine (left, $n=33$ ) or alcohol (right, $n=8$ ) functionalities (Tables S3 and S4). All metabolite concentrations were $100 \mathrm{ng} / \mathrm{mL}$. Derivatives were not observed for BC concentrations $<0.05 \mathrm{M}$. 
tives. $^{37}$ We generally observed $10-1000 \times$ higher raw peak areas for benzoylated amines (amides) than for benzoylated alcohols (esters) at the same metabolite concentration. This may be due to differences in derivatization efficiency, extraction efficiency, derivative solubility, or ionization efficiency. As a result, we prioritized method optimization for alcohols. At high concentrations, we often detected single and multilabeled derivatives for compounds with more than one derivatizable functional group (e.g., double-labeled DHPS; Figure S1), although the method was generally most sensitive for single-labeled compounds (Table 1) regardless of optimization parameters.

Derivatization Optimization. We focused on the following parameters for derivatization optimization: buffer type, reagent $(\mathrm{NaOH}$ and $\mathrm{BC})$ concentrations, and organic modifier addition. We compared MPs without a buffer ${ }^{34}$ and with tetraborate, ${ }^{39}$ phosphate, ${ }^{37}$ and carbonate buffers. ${ }^{40}$ All metabolites were detected in each treatment, and buffer presence did not drastically alter MP (Figure S4), possibly because seawater and saline culture media are naturally buffered by carbonate. We recommend omitting buffer in saline samples, but a buffer may improve result consistency in fresher water.

We tested the effect of dissolving $\mathrm{BC}$ in different organic solvents. Acetonitrile and methanol have been used to derivatize amine and phenolic alcohol metabolites ${ }^{40}$ and polyamines, ${ }^{31}$ respectively, although other studies added BC directly to aqueous samples without an organic solvent. ${ }^{34}$ Acetonitrile is not well miscible with seawater, so we compared methanol and acetone as organic modifiers. Despite large variability in MP for different metabolites, we observed a higher median MP when $\mathrm{BC}$ was dissolved in acetone than in methanol (Figure S5).

$\mathrm{BC}$ derivatization initiates under high $\mathrm{pH}$. As the reaction proceeds and protons accumulate, the $\mathrm{pH}$ decreases and nucleophiles are increasingly protonated, inhibiting further reaction. ${ }^{30}$ During benzoylation, BC reacts with water to form benzoic acid, which is insoluble in water when protonated $\left(\mathrm{p} K_{\mathrm{a}}\right.$ $=4.2$ ) and likely forms the observed white precipitate. We observed this precipitate under low $\mathrm{pH}(<4.2)$ and in the absence of organic solvents, which dissolve the precipitate. Precipitate formation occurred at an intermediate $\mathrm{NaOH}$ concentration and was coincident with efficient alcohol derivatization (Figure S6). Precipitation facilitates preextraction removal of excess reagent as benzoic acid, thereby reducing SPE overloading and accumulation on downstream instrumentation.

We determined the optimal MP with respect to all reagent $(\mathrm{NaOH}, \mathrm{BC}$, and acetone) concentrations and metabolite functionalities in seawater and spent medium (Figures 2 and S7; Table S2). In both matrices, MP was optimal at $\sim 0.1$ to 0.2 and 0.2 to $0.4 \mathrm{M} \mathrm{NaOH}$ for amine and alcohol derivatives, respectively, with a sharp decline in $\mathrm{MP}$ at higher $\mathrm{NaOH}$ concentrations ( $>0.6$ and $>0.4 \mathrm{M}$ for amine and alcohol derivatives, respectively). This trend was particularly pronounced for alcohol derivatives. At optimal $\mathrm{NaOH}$ concentrations, MP was stable with respect to $\mathrm{BC}$ concentrations between 50 and $250 \mathrm{mM}$ for alcohol- and amine-containing metabolites. MP was stable across acetone concentrations for alcohol functionalities but was slightly higher below $\sim 20$ to $30 \%$ acetone for amine derivatives in both matrices. We recommend reagent concentrations of $0.2 \mathrm{M} \mathrm{NaOH}, 15 \%$ acetone, and $70 \mathrm{mM} \mathrm{BC}$ for both sample types. These concentrations maximize MP while minimizing $\mathrm{BC}$ concentration and reducing benzoic acid formation.

Postderivatization Sample Prep. We evaluated preextraction acetone removal and sample acidification, as both influence derivative and benzoic acid solubilities and could improve PPL extraction efficiency. Upon acetone removal, the quantity of precipitate increased substantially, and we could remove a large fraction before SPE loading. Pre-extraction acetone removal increased the raw peak area of all metabolites up to $60 \times$ in seawater and $26 \times$ in spent medium (medians $=$ $2.4 \times$ and $2.1 \times$, respectively). The median MP did not vary greatly with $\mathrm{pH}$ (Figure S8), and derivatives were detected at all $\mathrm{pH}$ levels tested (1-5). Acetone removal and acidification likely increased extraction efficiency by reducing benzoic acid overloading on the resin, increasing analyte interactions with the solid phase relative to the liquid phase (acetone), and enhancing protonation of negatively charged groups, such as carboxylic acids. ${ }^{17}$

We recommend extracting 10 and $25 \mathrm{~mL}$ for culture and seawater samples, respectively, with a $1 \mathrm{~g}$ PPL cartridge (SText; Figures S9-S11). These sample volumes are slightly higher than volumes used for GC-MS analysis $(1 \mathrm{~mL})^{15}$ and $10-100 \times$ lower than those required for LCMS metabolite analysis without derivatization $(4-10 \mathrm{~L}) .{ }^{14}$ Our method thereby shortens the average field processing times over the PPL method ${ }^{14}$ from 60 to 5 min per sample, substantially increasing sample throughput. We determined the optimal reconstitution conditions by varying the $\mathrm{ACN}$ concentration added to a $1 \mathrm{~mL}$ sample, but we observed chromatographic peak splitting and tailing at CAN $>\sim 15$ to $20 \%$. Benzoylated metabolites were observed for all ACN concentrations, with substantial variability in MP across metabolites (Figure S12). In seawater, $\mathrm{MP}$ and $\mathrm{ACN}$ concentrations were not correlated; in spent medium, the median MP increased slightly with increasing $\mathrm{ACN}$ concentration. We recommend reconstituting samples by first washing the precipitate with $5 \% \mathrm{ACN} /$ water and adding $\mathrm{ACN}$ to a final concentration of $10 \%$ to prevent precipitation during storage.

UHPLC-ESI-MSMS. Benzoylation improved chromatographic retention of metabolites (Figure 3). Without derivatization, the majority of these metabolites elute early with poor chromatographic resolution, whereas their benzoyl derivatives eluted across the gradient with improved separation (Figure 3). Most derivatives exhibited sharp, well-resolved peaks (Figure S13), although peak tailing was observed for some, particularly early-eluting, phosphate-containing metabolites such as glucosamine-6-phosphate. We recommend monitoring the peak area drift during sample runs using the SIL-IS peak area (Figure S14). A benzoic acid peak was observed at $7.5 \mathrm{~min}$ (Figure S15).

Method Accuracy. We improved method accuracy through the use of SIL-ISs and evaluated the effect of matrix-specific biases using process effect (PE). Consistent with previous work, the ${ }^{13} \mathrm{C}_{6}$ SIL-ISs outperformed $\mathrm{D}_{5}$ SIL-ISs (Table S6), and SIL-IS ion suppression/enhancement of or by the analytes was minimal except at very high concentrations ( $\geq 30000 \mathrm{pg} / \mathrm{mL}$; Figure S2, S-Text). We compared calibration curves prepared with and without normalization to the SIL-IS. For most derivatives, calibration curves were improved (increased $R^{2}$ ) by normalization of the raw peak areas to the SIL-IS peak areas (Figures S16 and S17). For example, of 52 compounds detected with negative polarity, the calibration curves for 33 and 40 compounds were acceptable 

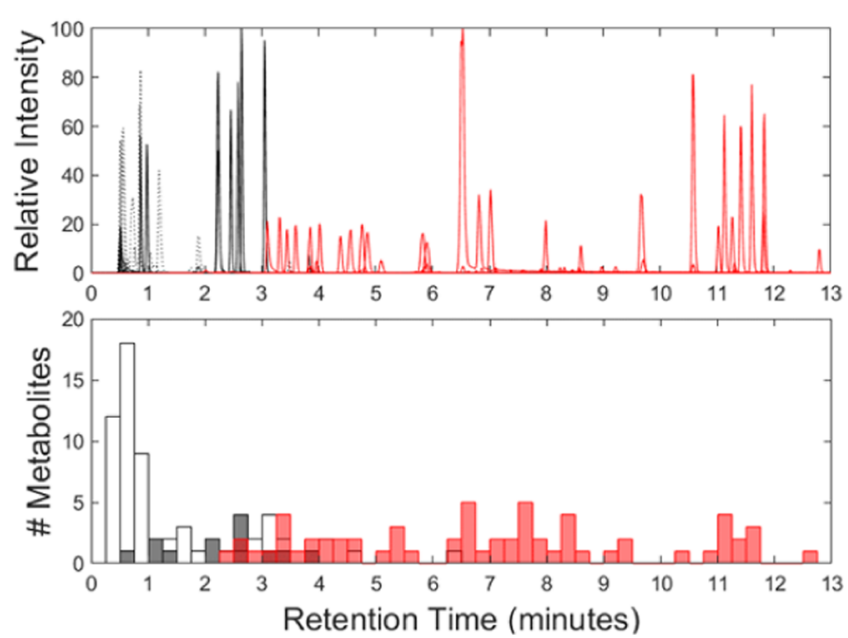

Figure 3. Composite extracted ion chromatograms (EICs; top) and retention times (bottom) of representative metabolites. Red lines and bars represent metabolites that were spiked into seawater, benzoylated, and extracted $(n=52)$. Dotted lines and open bars are the same metabolites in DI that were not derivatized or extracted, while black lines and gray bars represent the subset that can be PPLextracted from seawater without derivatization $(n=13)$. Concentrations were $100 \mathrm{ng} \mathrm{mL}^{-1}$. No data were recorded during the initial $0.5 \mathrm{~min}$ (solvent front) in the top panel. Histogram bins are $0.25 \mathrm{~min}$.

for quantification in seawater and spent medium, respectively, as compared to only 13 and 26 compounds prepared from raw peak areas.

PEs were determined in the two matrix types, where $\mathrm{PE}$ indicates enhancement $(+\mathrm{PE})$ or reduction $(-\mathrm{PE})$ of the signal relative to a reference matrix, and a PE of zero indicates no matrix-specific bias. PEs calculated from raw peak areas reflect biases of the entire process, while PEs calculated from SIL-IS normalized peak areas reflect biases in only the derivatization step. For oligotrophic seawater, we calculated PE for samples from four BATS depths (0,95, 160, $200 \mathrm{~m} ; n=6$ per curve) and DI water $(n=12)$ as compared to BATS deep water $(1000$ $\mathrm{m} ; n=12$; Table S3). For cultures, we calculated PE for spent media from $T$. pseudonana and $R$. pomeroyi cultures relative to the axenic medium blanks ( $\mathrm{L} 1$ and $f / 2+$ propionate; Table S4). Fewer compounds could be quantified in DI water than in seawater because these compounds were either not derivatized in DI water or the DI standard curve was inferior (Figure S16 and Table S3). This resulted in only 54 quantifiable compound/polarity pairs (both polarities) for SIL-IS normalized peak areas in DI water compared to 66 in seawater.

In both seawater and culture media, the median PE for all treatments deviated farther from 0 when calculated from raw peak areas ( -28 and $17 \%$, respectively) than from SIL-IS normalized peak areas (4.1 and 0.3\%; Figures S16 and S17; Tables S3 and S4), indicating that the majority of matrixspecific biases occur downstream of derivatization. In seawater, the PEs for raw peak areas were more negative at shallower depths than at deeper depths and in DI water (Figure S16), indicating relative signal depression in shallow water. This trend may be driven by labile organic matter content, which is higher in shallower, more biologically productive, water than in deep and DI water (Table S7). Organic matter may interfere with extraction or derivative solubility. In both matrix types, the $\mathrm{PE}$ of some compounds fell outside the acceptable range $( \pm 20 \%)$. Because PE represents a systematic bias, rather than a random error, the concentrations of these metabolites should be interpreted cautiously. Overall, normalization to the analytespecific SIL-IS increases the number of quantifiable compounds and reduces the likelihood of systemic biases due to matrix effects.

Method Validation. We compared quantification in five samples using either a matrix-matched calibration curve prepared in freshly collected deep seawater $(1000 \mathrm{~m}$ at BATS) or the standard addition method (SAM) with both raw and SIL-IS normalized peak areas. We chose deep ocean water because it should have the lowest background of dissolved metabolites and organic carbon (Table S7) with similar $\mathrm{pH}$ and ionic strength as shallower water. Indeed, many metabolite concentrations in deep water were lower than those in the shallower depths, although some were still abundant (Table S8). At the shallow depths, calculated metabolite concentrations were similar using the two methods, but the relative standard deviation (RSD) was much higher for concentrations calculated using the SAM, reflecting the higher inherent error of the $x$-intercept relative to a point within the bounds of the calibration curve. ${ }^{45}$

For example, DHPS concentrations calculated from the SILIS normalized matrix-matched curve were $0.10 \pm 0.01$ and 0.35 $\pm 0.02 \mathrm{nM}$ at 1 and $95 \mathrm{~m}$ (RSD $=13$ and $4.4 \%$, respectively). Using the SAM, DHPS concentrations were $0.12 \pm 0.07$ and $0.21 \pm 0.11 \mathrm{nM}$ at 1 and $95 \mathrm{~m}$, respectively (RSD $=62$ and $51 \%)$.

Each method contains a source of inaccuracy: PE in the matrix-matched curve and extrapolation of the $x$-intercept below the known linear range of the calibration curve in the SAM. $^{23}$ The SAM also suffers from low precision (RSD) ${ }^{23}$ and practical limitations. We used a 6-point curve for the SAM, but in practice, analysts generally use three or four standard additions, optimally with concentrations equal to $1 \times, 2 \times, 3 \times$, and $4 \times$ the analyte. ${ }^{45}$

Method Sensitivity. Most of our data fell into the case of homoscedastic standard deviation, enabling DL calculations with the method of Vogelgesang and Hädrich. ${ }^{50}$ The calculation for the heteroscedastic case ${ }^{48}$ generally resulted in a lower DL because it extrapolates below the range of standard deviations observed, rather than using the aggregate standard deviation. Although both calculations minimize biases associated with a nonzero background analyte concentration, as compared to the EPA calculation (LOD), ${ }^{47}$ they do not entirely eliminate this bias, particularly if the background concentration is high. This is especially true for the homoscedastic case, where the DL is derived from the aggregate standard deviation for all standard concentrations. Some analyte background concentrations were elevated in the calibration matrices, particularly in culture media, which likely resulted in an overestimation of the DLs. However, because of the poor derivative recovery in DI water (Figure S16 and Table S3), the "zero" background signal cannot be determined.

Our method is more sensitive in oligotrophic seawater than in cultures. DLs ranged from 0.010 to $2 \mathrm{nM}$ for oligotrophic seawater (median $0.079 \mathrm{nM}$ ) and 0.081 to $150 \mathrm{nM}$ for culture media (median $2.3 \mathrm{nM}$; Table 1). This disparity may result from differences in sample volume or type and the more conservative calculation for cultures. While culture media and seawater have similar salinity and $\mathrm{pH}$, DOC concentrations are substantially higher in culture media than in oligotrophic seawater (Table S8), and the DOC character also differs. DOC concentration and character may impact derivatization and extraction ${ }^{16}$ efficiencies. For example, phytoplankton release 
alcohol-containing polysaccharides, ${ }^{13}$ which may compete with metabolites for BC or adhere to the PPL, thereby lowering analyte retention.

Some compounds quantified by our method are also targeted by the PPL method, ${ }^{16}$ the GC-MS method, ${ }^{15}$ and the $o$-phthalaldehyde (OPA) amino acid method. ${ }^{11}$ Our seawater DLs are similar in magnitude to those obtained with underivatized PPL extraction $(0.007-2.4 \mathrm{nM})^{16}$ and lower than those reported using a GC-MS derivatization method $(60-1000 \mathrm{nM}){ }^{15}$ The DLs we report for culture media are of similar magnitude to those from Sogin et al. ${ }^{15}$ The seawater DLs for the 22 amino acids reported here (0.020-0.24 nM) are lower than those of the OPA method $(\sim 0.5$ to $1 \mathrm{nM}),{ }^{11}$ while the amino acid DLs for cultures are higher $(0.12-87 \mathrm{nM})$, likely due to the conservative nature of that calculation.

The DLs reported here were sufficiently sensitive to quantify numerous metabolites in culture media and at BATS (Table S8). Additional benzoylated metabolites were identified but did not meet the quality threshold for quantification (Table S8), particularly in culture media. These will likely be quantifiable after future method refinements.

\section{CONCLUSIONS}

Our pre-extraction benzoylation method is sensitive and robust for the detection and quantification of a broad suite of amineand alcohol-containing dissolved metabolites in seawater and saline culture media. DLs are nano- to picomolar, and matrix biases are minimal due to the use of matrix-matched calibration curves and analyte-matched SIL-ISs. This method quantifies numerous compounds that have not been previously measured in seawater, limiting the understanding of their role in aquatic systems. For example, sulfonates such as DHPS and isethionate have emerged as key players in marine ecosystems and the sulfur cycle, ${ }^{4}$ but, using the existing methodology, these compounds cannot be extracted from saline solution ${ }^{16}$ without derivatization. Our method, which is sensitive and precise for both compounds, will facilitate an improved understanding of these processes and others in laboratory studies and in the field.

\section{ASSOCIATED CONTENT}

\section{s) Supporting Information}

The Supporting Information is available free of charge at https://pubs.acs.org/doi/10.1021/acs.analchem.0c03769.

Supporting Text and Figures S1-S17 (PDF)

Tables S1-S9 (XLSX)

\section{AUTHOR INFORMATION}

\section{Corresponding Author}

Brittany Widner - Department of Marine Chemistry and Geochemistry, Woods Hole Oceanographic Institution, Woods Hole, Massachusetts 02543, United States; 이이.org/ 0000-0002-1527-2717; Email: brittany.widner@ gmail.com

\section{Authors}

Melissa C. Kido Soule - Department of Marine Chemistry and Geochemistry, Woods Hole Oceanographic Institution, Woods Hole, Massachusetts 02543, United States
Frank Xavier Ferrer-González - Department of Marine Sciences, University of Georgia, Athens, Georgia 30602, United States

Mary Ann Moran - Department of Marine Sciences, University of Georgia, Athens, Georgia 30602, United States; (1) orcid.org/0000-0002-0702-8167

Elizabeth B. Kujawinski - Department of Marine Chemistry and Geochemistry, Woods Hole Oceanographic Institution, Woods Hole, Massachusetts 02543, United States; (1) orcid.org/0000-0001-8261-971X

Complete contact information is available at:

https://pubs.acs.org/10.1021/acs.analchem.0c03769

\section{Notes}

The authors declare no competing financial interest.

\section{ACKNOWLEDGMENTS}

The authors thank Craig Carlson, Shuting Liu, and the BIOSSCOPE team for DOC data and facilitating BATS sampling. Noah Germolus, Erin McParland, Krista Longnecker, and Gretchen Swarr provided thoughtful conversations and edits to manuscript drafts. K. Longnecker provided culture DOC data. Funding included the Gordon and Betty Moore Foundation (Award GBMF5503 to M.A.M. and E.B.K.), Simons Foundation International (Award 409923 to E.B.K.), and the National Science Foundation (Award 1656311 to M.A.M.).

\section{REFERENCES}

(1) Moran, M. A.; Kujawinski, E. B.; Stubbins, A.; Fatland, R.; Aluwihare, L. I.; Buchan, A.; Crump, B. C.; Dorrestein, P. C.; Dyhrman, S. T.; Hess, N. J.; et al. Proc. Natl. Acad. Sci. U.S.A. 2016, 113, 3143-3151.

(2) Heal, K. R.; Carlson, L. T.; Devol, A. H.; Armbrust, E. V.; Moffet, J. W.; Stahl, D. A.; Ingalls, A. E. Rapid Commun. Mass Spectrom. 2014, 28, 2398-2404.

(3) Sañudo-Wilhelmy, S. A.; Gómez-Consarnau, L.; Suffridge, C.; Webb, E. A. Annu. Rev. Mar. Sci. 2014, 6, 339-367.

(4) Moran, M. A.; Durham, B. P. Nat. Rev. Microbiol. 2019, 17, 665678

(5) Sipler, R. E.; Bronk, D. A. Biogeochemistry of Marine Dissolved Organic Matter; Hansell, D. A.; Carlson, C. A., Eds.; Academic Press, 2015; pp 127-232.

(6) van Tol, H. M.; Amin, S. A.; Armbrust, E. V. ISME J. 2017, 11, $31-42$.

(7) Gram, L.; Grossart, H.-P.; Schlingloff, A.; Kiørboe, T. Appl. Environ. Microbiol. 2002, 68, 4111-4116.

(8) Fiore, C. L.; Longnecker, K.; Kido Soule, M. C.; Kujawinski, E. B. Environ. Microbiol. 2015, 17, 3949-3963.

(9) Azam, F.; Fenchel, T.; Field, J. G.; Gray, J. S.; Meyer-Reil, L. A.; Thingstad, F. Mar. Ecol.: Prog. Ser. 1983, 10, 257-263.

(10) Nagata, T. Microbial Ecology of the Oceans; Kirchman, D. L., Ed.; Wiley-Liss: New York, 2000; pp 121-152.

(11) Mopper, K.; Lindroth, P. Limnol. Oceanogr. 1982, 27, 336-347.

(12) Suárez-Suárez, A.; Tovar-Sánchez, A.; Rosselló-Mora, R. Anal. Chim. Acta 2011, 701, 81-85.

(13) Panagiotopoulos, C.; Sempéré, R. Limnol. Oceanogr.: Methods 2005, 3, 419-454.

(14) Kido Soule, M. C.; K, L.; W M, J.; Kujawinski, E. B. Mar. Chem. 2015, 177, 374-387.

(15) Sogin, E. M.; Puskas, E.; Dubilier, N.; Liebeke, M. mSystems 2019, 4, 1-14.

(16) Johnson, W. M.; Kido Soule, M. C.; Kujawinski, E. B. Limnol. Oceanogr.: Methods 2017, 15, 417-428.

(17) Dittmar, T.; Koch, B.; Hertkorn, N.; Kattner, G. Limnol. Oceanogr.: Methods 2008, 6, 230-235. 
(18) Longnecker, K.; Soule, M. C. K.; Kujawinski, E. B. Mar. Chem.

2015, 168, 114-123.

(19) Becker, J. W.; Berube, P. M.; Follett, C. L.; Waterbury, J. B.; Chisholm, S. W.; DeLong, E. F.; Repeta, D. J. Front. Microbiol 2014, 5, $1-14$.

(20) Annesley, T. M. Clin. Chem. 2003, 49, 1041-1044.

(21) Hewavitharana, A. Crit. Rev. Anal. Chem. 2009, 39, 272-275.

(22) Boysen, A. K.; Heal, K. R.; Carlson, L. T.; Ingalls, A. E. Anal. Chem. 2018, 90, 1363-1369.

(23) Andersen, J. E. TrAC, Trends Anal. Chem. 2017, 89, 21-33.

(24) Tan, A.; Boudreau, N.; Lévesque, A. LC-MS in Drug Bioanalysis; Springer, 2012; pp 1-32.

(25) Han, W.; Li, L. Clinical Metabolomics; Springer, 2018; pp 213225.

(26) Barnaby, O. S.; Benitex, Y.; Cantone, J. L.; McNaney, C. A.; Olah, T. V.; Drexler, D. M. Bioanalysis 2015, 7, 2501-2513.

(27) Qi, B.-L.; Liu, P.; Wang, Q.-Y.; Cai, W.-J.; Yuan, B.-F.; Feng, Y.Q. TrAC, Trends Anal. Chem. 2014, 59, 121-132.

(28) Guo, K.; Li, L. Anal. Chem. 2009, 81, 3919-3932.

(29) Escrig-Doménech, A.; Simó-Alfonso, E.; Herrero-Martínez, J.; Ramis-Ramos, G. J. Chromatogr. A 2013, 1296, 140-156.

(30) Redmond, J. W.; Tseng, A. J. Chromatogr. A 1979, 170, 479481.

(31) Asotra, S.; Mladenov, P. V.; Burke, R. D. J. Chromatogr. A 1987, 408, 227-233.

(32) Liu, R.; Jia, Y.; Cheng, W.; Ling, J.; Liu, L.; Bi, K.; Li, Q. Talanta 2011, 83, 751-756.

(33) Gupta, R. N.; Eng, F.; Gupta, M. L. Clin. Chem. 1982, 28, 3233.

(34) Holčapek, M.; Virelizier, H.; Chamot-Rooke, J.; Jandera, P.; Moulin, C. Anal. Chem. 1999, 71, 2288-2293.

(35) Kuribayashi, M.; Tsuzuki, M.; Sato, K.; Abo, M.; Yoshimura, E. Chromatographia 2008, 67, 339-341.

(36) Nordström, A.; Tarkowski, P.; Tarkowska, D.; Dolezal, K.; Åstot, C.; Sandberg, G.; Moritz, T. Anal. Chem. 2004, 76, 2869-2877.

(37) Oehlke, J.; Brudel, M.; Blasig, I. E. J. Chromatogr. B: Biomed. Sci. Appl. 1994, 655, 105-111.

(38) Miyagi, M.; Yokoyama, H.; Hibi, T. J. Chromatogr. B 2007, 854, 286-290.

(39) Song, P.; Mabrouk, O. S.; Hershey, N. D.; Kennedy, R. T. Anal. Chem. 2012, 84, 412-419.

(40) Wong, J.-M. T.; Malec, P. A.; Mabrouk, O. S.; Ro, J.; Dus, M.; Kennedy, R. T. J. Chromatogr. A 2016, 1446, 78-90.

(41) Mishra, S.; Singh, V.; Jain, A.; Verma, K. K. Analyst 2001, 126, $1663-1668$.

(42) Tran, B. N.; Okoniewski, R.; Bucciferro, A.; Jansing, R.; Aldous, K. M. J. AOAC Int. 2014, 97, 232-237.

(43) Henderson, C. M.; Shulman, N. J.; MacLean, B.; MacCoss, M. J.; Hoofnagle, A. N. Clin. Chem. 2018, 64, 408-410.

(44) Pino, L. K.; Searle, B. C.; Bollinger, J. G.; Nunn, B.; MacLean, B.; MacCoss, M. J. Mass Spectrom. Rev. 2020, 39, 229-244.

(45) Danzer, K.; Currie, L. A. Pure Appl. Chem. 1998, 70, 993-1014.

(46) Currie, L. A. Anal. Chem. 1968, 40, 586-593.

(47) Boyd, R. K.; Basic, C.; Bethem, R. A. Trace Quantitative Analysis by Mass Spectrometry; John Wiley \& Sons, 2011.

(48) Zorn, M. E.; Gibbons, R. D.; Sonzogni, W. C. Environ. Sci. Technol. 1999, 33, 2291-2295.

(49) Griffith, D. R.; Kido Soule, M. C.; Matsufuji, H.; Eglinton, T. I.; Kujawinski, E. B.; Gschwend, P. M. Environ. Sci. Technol. 2014, 48, $2569-2578$

(50) Vogelgesang, J.; Hädrich, J. Accredit. Qual. Assur. 1998, 3, 242255.

(51) Chawla, S.; Patel, H. K.; Gor, H. N.; Vaghela, K. M.; Solanki, P. P.; Shah, P. G. J. AOAC Int. 2017, 100, 616-623. 